\title{
Cubic Nonlinear Optical Effects in Conjugated Polymers
}

\author{
F. KAJZAR and J. Messier \\ C.E.A.-I.R.D.I., Centre d'Etudes Nucléaires de Saclay, \\ Département d'Electronique et d'Instrumentation Nucléaire, \\ Laboratoire d'Etudes et de Recherches Avancées, \\ 91191 GIF-SUR-YVETTE CEDEX, France
}

(Received October 13, 1986)

\begin{abstract}
A general survey of harmonic generation techniques in polymer thin film is given and some recent results of wave dispersed third harmonic generation measurements on conjugated polymers with one dimensional $\pi$-electron delocalization are reviewed and discussed. These polymers exhibit very large cubic hyperpolarizabilities in near infrared. Some multiphoton (two and three photon) resonance enhancements in cubic susceptibility are observed. The two photon level with the same symmetry as the fundamental one lies in studied polymers below the allowed for optical transition one photon level. Possible practical applications of observed resonances are discussed.
\end{abstract}

KEY WORDS Conjugated Polymers / Polymer Thin Films / Nonlinear Susceptibility / Nonlinear Refractive Index / Resonance Effects / Two Photon Absorption /

The rapidly developping optical telecommunications, image processing and perspectives of design of optical computing systems call the roll to highly efficient, with fast response time, nonlinear optical materials. In recent years it was shown that the organic noncentrosymmetric materials, with internal charge transfer, have about two order of magnitude higher second order susceptibilities compared to the most representative their inorganic counterpart lithium niobate.

The emergence of conjugated one-dimensional polymers has shown a new way of getting highly efficient third order nonlinear materials which may found an application in a large variety of optical devices: bistables, dynamical memories, phase conjugation, parametric amplification, four wave mixing, Raman scattering etc. In fact, the theoretical calculations of Rustagi and Ducuing ${ }^{1}$ and Agrawal et al. ${ }^{2}$ show that such conjugated one dimensional $\pi$ electron systems have large cubic susceptibilities depending strongly on delocalization length $L\left(\chi^{(3)} \sim L^{\alpha}, \alpha=5 \div 6\right)$.

The conjugated one dimensional organic polymers possess also some very interesting physico-chemical and mechanical properties. They are mechanically very stable, with large value of Young modulus in polymer chain direction, comparable to that of $3 \mathrm{~d}$ metals. ${ }^{3,4}$ Some of these polymers, polydiacetylenes, polymerize in solid state, giving locally large linear refractive index changes. This property can be used in integrated optical circuits design. Polymers are also suitable materials for thin film deposition. This can be obtained directly from monomer thin film deposition (Langmuir-Blodgett technique, ${ }^{5}$ epitaxy, ${ }^{6}$ shear method ${ }^{6}$ etc.) and subsequent polymerization or polymer thin film deposition: e.g., Shirakawa's technique of polyacetylene thin film deposition. In the case of soluble polymers very simple thin film deposition techniques can be used: spinning, dipping, solvent evapora- 
tion, etc.

Due to the large cubic susceptibility of conjugated polymers their nonlinearity can be studied on relatively small interaction lengths. The simplest and direct methods of cubic nonlinearities measurements are harmonic light generation techniques: third harmonic generation (THG) and electric field induced second harmonic generation (EFISHG). Both techniques can be done by transmission. Moreover, due to small polymer film thickness, the measurements can be done in polymer absorption range for harmonic frequency giving access to forbidden for one photon transition levels.

In this paper we give a survey of harmonic light generation techniques in thin films and we review some results obtained for conjugated polymers for cubic susceptibilities together with observed multiphoton resonances. Some practical aspects of these resonances will be also discussed.

\section{THEORY}

\section{Nonlinear Polarization of a Dielectric and Har- monic Generation}

The polarization of a dielectric can be expanded in dipolar approximation in the external electric field $E$ power series

$$
P=\chi^{(1)}: E+\chi^{(2)}: E E+\chi^{(3)}: E E E+\ldots
$$

where $\chi^{(n)}$ are $(n+1)$ rank tensors. If there exists a spontaneous internal polarization then $E$ is an effective electric field experienced by molecules.

The tensor $\chi^{(1)}$ in eq 2 describes linear optical properties whereas higher rank tensors describe nonlinear processes. For centrosymmetric structures like conjugated polymers with one dimensional delocalization $\chi^{(2)} \equiv 0$. The tensor $\chi^{(3)}$ describes cubic nonlinear processes like: THG, EFISHG, four wave mixing (FWM), optical bistability, optical Kerr effect etc. For a conjugated linear polymer chain the $\chi^{(3)}{ }_{x x x x}$ tensor component is most enhanced.
For the nonlinear processes interesting us the nonlinear polarization components are given by

$$
\begin{aligned}
P_{x}^{\mathrm{NL}}= & \frac{1}{4} \chi_{x x x x}^{(3)}(-3 \omega ; \omega, \omega, \omega)\left[E_{x}^{\omega}\right]^{3}-\mathrm{THG} \\
P_{x}^{\mathrm{NL}}= & \frac{3}{2} \chi_{x x x x}^{(3)}(-2 \omega ; \omega, \omega, o)\left[E_{x}^{\omega}\right]^{2} E_{o} \\
& - \text { EFISHG }
\end{aligned}
$$

where the DC external field $E_{\mathrm{o}}$ is applied parallel to the incident light polarization.

\section{Harmonic Light Generation}

For a nonlinear slab with thickness $l$ immersed between two linear media the output harmonic field is given by ${ }^{6}$

$$
E_{\mathrm{H}}=A \mathrm{e}^{i \psi_{\mathrm{H}}}\left[\mathrm{e}^{i \Delta \psi}-1\right]
$$

where

$$
\Delta \psi=\psi_{\mathbf{F}}-\psi_{\mathbf{H}}
$$

is phase mismatch between fundamental (F) and harmonic $(\mathrm{H})$ wave in medium with

$$
\psi_{\mathrm{F}(\mathrm{H})}=3 \omega n_{\omega_{\mathrm{F}(\mathrm{H})}} \cos \theta_{\omega_{\mathrm{F}(\mathbf{H})}} l c
$$

where $n_{\omega_{\mathbf{F}(\mathbf{H})}}$ are refractive indices and $\theta_{\omega_{\mathbf{F}(\mathbf{H})}}$ propagation angles.

$A$ in eq 5 is a factor arising from boundary conditions and for THG

$$
A=\pi \frac{\chi^{(3)}}{\Delta \varepsilon} A_{1}\left(t_{12}^{\omega} E_{\omega}\right)^{3}
$$

where $\Delta \varepsilon$ is dielectric constant dispersion, $t_{12}^{\omega}$ is transmission factor

$$
t_{12}^{\omega}=\frac{2 n_{\omega}^{1} \cos \theta_{\omega}^{1}}{n_{\omega}^{1} \cos \theta_{\omega}^{1}+n_{\omega}^{2} \cos \theta_{\omega}^{2}}
$$

and $A_{1}$ is a factor arising from boundary conditions

$$
A_{1}=\frac{n_{\omega_{F}}^{2} \cos \theta_{\omega_{F}}^{2}+n_{\omega_{H}}^{3} \cos \theta_{\omega_{H}}^{3}}{n_{\omega_{H}}^{2} \cos \theta_{\omega_{H}}^{2}+n_{\omega_{H}}^{3} \cos \theta_{\omega_{H}}^{3}}
$$

Superscripts in eq 9 and 10 refer to corresponding media (1-input, 2-nonlinear, 3-out- 
put).

Equation 5 is an approximation for harmonic field ( $c f$. Kajzar and $\mathrm{Messier}^{8}$ ). It holds when the refractive indices of nonlinear medium are not very large as it is the case of organic matter. It takes into account all reflected harmonic waves. We note here that the simplifications used often in getting harmonic field expression and consisting on a neglect of all reflected waves from interfaces other than that under consideration ${ }^{9-11}$ lead to nonsensical results in the case of thin films and is demonstrated by e.g., an independence of reflected harmonic wave intensity on thin film thickness (see discussion in Kajzar and Messier $^{12}$ ).

For a serie of nonlinear media as it is the case of thin films deposited on a substrate whose nonlinear response not always can be neglected with respect to that of the film itself, the resultant harmonic field is a superposition of fields generated in each medium separately

$$
E_{\mathrm{H}}^{\mathrm{R}}=E_{\mathrm{H}}^{1} T_{1}+E_{\mathrm{H}}^{2} T_{2}+\ldots
$$

where $T$ 's are whole transmission factors.

One can distinct two special cases:

i) Thin films deposited on one side of substrate. In this case the outgoing harmonic intensity is given by

$$
\begin{aligned}
I_{3 \omega}(\theta)= & \frac{64 \pi^{4}}{c^{2}} \mid \mathrm{e}^{i\left(\psi_{\omega}^{s}+\psi_{3 \omega}^{p}\right)}\left(\frac{\chi^{(3)}}{\Delta \varepsilon}\right)_{s} \\
& \times\left[A_{1}^{s} T_{1}\left(1-\mathrm{e}^{-i \Delta \psi^{s}}\right)\right. \\
& \left.+\rho T_{2} A_{1}^{p}\left(E^{i \Delta \psi^{p}}-1\right)\right]\left.\right|^{2}\left(I_{\omega}\right)^{3}
\end{aligned}
$$

where superscripts or subscripts $\mathrm{s}$ and $\mathrm{p}$ refer to substrate and polymer film, respectively, and

$$
\rho=\left(\chi^{(3)} / \Delta \varepsilon_{p} /\left(\chi^{(3)} / \Delta \varepsilon\right)_{s}\right.
$$

In a general case $\rho$ may be complex: $\rho=|\rho| \mathrm{e}^{i \phi}$, as well as complex are all transmission factors $T$ 's and $A_{1}$ s coefficients in an absorbing me- dium for fundamental and/or harmonic frequency.

ii) Thin films deposited on both sides of substrate. The resultant harmonic field is given in this case by

$$
\begin{aligned}
I_{3 \omega}= & \frac{64 \pi^{4}}{c^{2}} \mid\left(\frac{\chi^{(3)}}{\Delta \varepsilon}\right)_{s} \mathrm{e}^{i \psi} \rho T_{1} A_{1}^{p}\left(1-\mathrm{e}^{-i \Delta \psi_{p}}\right) \\
& +T_{2} A_{1}^{s}\left(\mathrm{e}^{i \Delta \psi_{s}}-1\right) \\
& +\left.\rho T_{3} A^{\prime}{ }_{1}^{p} \mathrm{e}^{i \Delta \psi_{s}}\left(\mathrm{e}^{i \Delta \psi_{p}}-1\right)\right|^{2}\left(I_{\omega}\right)^{3}
\end{aligned}
$$

where

$$
\psi=\psi_{\mathrm{F}}^{p}+\psi_{\mathrm{H}}^{p}+\psi_{\mathrm{H}}^{s}
$$

Both eq 12 and 14 lead to complex interference spectra. They show a possibility of polymer susceptibility phase $\phi$ determination if that of substrate is known (e.g., $\chi_{\mathrm{s}}^{(3)}$ real as it is the case far of absorption bands and far from forbidden transitions). In the case of transparent thin films with thickness $l$ much smaller than the coherence length $l_{\mathrm{c}}=\lambda / 6|\Delta n|$ the term $\left(\mathrm{e}^{i \Delta \psi} p-1\right)$ is directly proportional to the polymer film thickness ${ }^{13}$ and does not depend upon $l_{\mathrm{c}}$.

We note here that eq 12 and 14 are true for THG measurement in vacuum. In air two additional nonlinear media are to be added (air before and after sample), what in a general case lead to much more complicated situation. ${ }^{8}$ In the case of EFISHG eq 5 and 6 are valid with $\mathrm{F}=\omega, \mathrm{H}=2 \omega$ and $A$ given by

$$
A=6 \pi \frac{\chi^{(3)}(-2 \omega ; \omega, \omega, o)}{\Delta \varepsilon} A_{1}\left(t_{12}^{\omega} E_{\omega}\right)^{2} E_{o}
$$

and for a thin film deposited on a solid substrate with electrodes one gets (for details see Chollet et al. ${ }^{14}$ ).

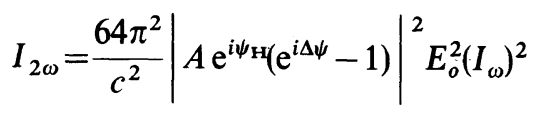




\section{EXPERIMENTAL}

\section{Apparatus}

A typical experimental arrangement for wave dispersed harmonic generation measurements is shown in Figure 1. The light source is a $Q$-switched Neodymium doped Yttrium Aluminium Garnet (Nd: YAG) laser operating at $1.064 \mu \mathrm{m}$ fundamental wavelength with pulse duration of $13 \mathrm{~ns}$ and repetition rate of $10 \mathrm{~Hz}$. The fundamental beam, after a frequency doubling in a phase matched KDP single crystal, is pumping a dye laser. The tunable in visible laser beam is subsequently shifted to near IR by stimulated Raman scattering (first and second Stoke radiation) in a high pressure hydrogen cell (56 bars). A Pellin-Broca prism allows to separate different outgoing wavelengths. With a corresponding choice of organic dyes one can cover $0.56 \div 1.9 \mu \mathrm{m}$ wavelength range. The parasit light is filtered by a fitter assembly and the chosen wavelength is focused on sample placed in a vacuum cell with fused silica windows. Again, the fundamental light is eliminated by corresponding filters and by a monochromator (this two step filtering is necessary in view of low conversion rate and parasit effects). The PMT signal is integrated in boxcar integrator. Rotation and/or translation of sample is controlled by a minicomputer which stockes also the harmonic intensity in function of the sample position. The possible fluctuations of the fundamental beam are monitored by a rapid photodiode. In THG experiments the measured hyperpolaritability is calibrated with a fused silica plate (Suprasil I) with known $\chi^{(3)}$ value $\left(\chi^{(3)}\right.$ $(-3 \omega ; \omega, \omega, \omega)=2.8 \times 10^{-14}$ e.s.u. $\left.{ }^{11}\right)$ and in EFISHG with a wedged $\alpha$-quartz single crystal $\left(\chi_{x x x}^{(2)}(2 \omega ; \omega, \omega)=1.2 \times 10^{-9}\right.$ e.s.u $\left.u^{9,15}\right)$.

\section{Thin Film Deposition}

The most of spectroscopie studies have been

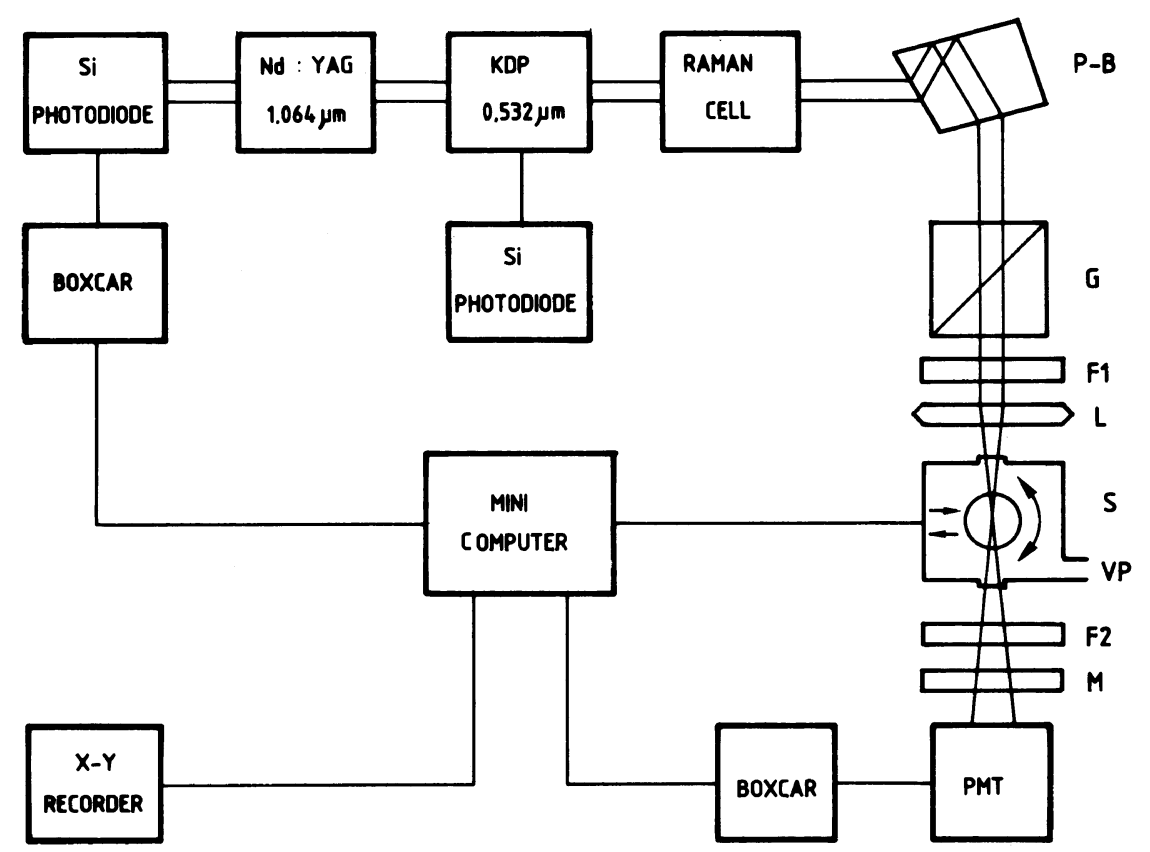

Figure 1. Schematic representation of experimental arrangement for wave dispersed harmonic generation measurements: P-B, Pellin-Broca prism; G, Glan polarizer; F, filters; L, lens; S, sample, VP, vacuum pump; M, monochromator; PMT, photomultiplier tube. 
done on polydiacetylene Langmuir-Blodgett multilayers. The Langmuir-Blodgett technique, diagrammatized in Figure 2, allows to get regular and oriented thin films with well controlled thickness starting from about 30 A (one monolayer) to a few thousands of angstroms. The used diacetylene monomer with chemical formula:

$$
\mathrm{CH}_{3}-\left(\mathrm{CH}_{2}\right)_{15}-\mathrm{C} \equiv \mathrm{C}-\mathrm{C} \equiv \mathrm{C}-\left(\mathrm{CH}_{2}\right)_{8}-\mathrm{COOH}
$$

forms a stable solid film on water subphase $^{5,16,19}$ with $5 \times 10^{-4} \mathrm{M} \mathrm{CdCl}_{2}$. This film can be directly polymerized on water surface ${ }^{17}$ with UV irrradiation and transferred as polymer film on a solid substrate (e.g., fused silica), or transferred in monomer form and subsequently polymerized. ${ }^{18}$ In the present case the second technique was used ( $c f$. Figure $2)$. The resulting polymer has following formula: $\quad \mathrm{CH}_{3}-\left(\mathrm{CH}_{2}\right)_{15}-\mathrm{C}-\mathrm{C} \equiv \mathrm{C}-\mathrm{C}-\left(\mathrm{CH}_{2}\right)_{8}-$ COOCd. $^{1 / 2}$

The LB multilayers can be characterized by a variety of spectroscopic methods (UV, visible, IR, crossed polarizers) and their orientation can be checked out by linear dichroism, ${ }^{20,21}$ electron microscopy, ${ }^{19} \mathrm{ESR}^{21}$ (if some magnetic ions are included into the matrix, e.g., $\mathrm{Cu}^{2+}$ in place of $\mathrm{Cd}^{2+}$ ) and $\mathrm{X}$ Ray $^{22}$ electron $^{23}$ or neutron ${ }^{24}$ diffraction

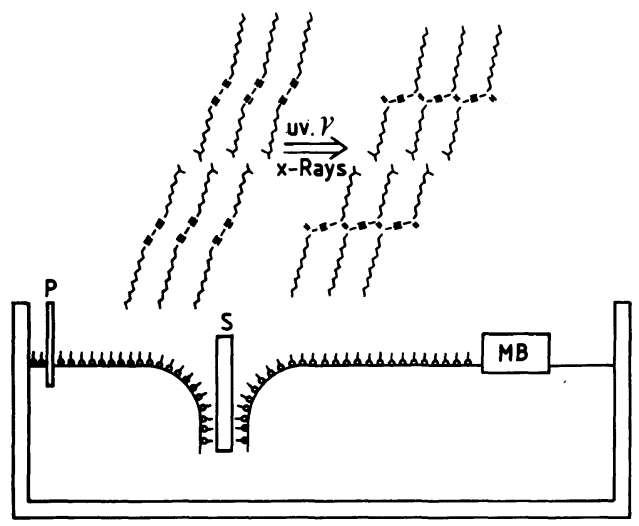

Figure 2. Principle of Langmuir-Blodgett thin film deposition. The upper part of figure show a polydiacetylene monomer (left) and polymer (right) bilayer: P, pressure sensor; $\mathrm{MB}$, mobile barrier; $\mathrm{S}$, substrate. techniques.

\section{RESULTS}

The THG measurements have been carried out by rotation of substrate with polymer film along an axis perpendicular to the light propagation direction. In Figure 3 we have shown some typical $\mathrm{TH}$ intensity spectra in function of incidence angle for 3 distinct cases: polymer film with a large value of $\chi^{(3)}$ on one side of substrate only (a blue form of PDA LB film), the same but with smaller value of $\chi^{(3)}$ (a polysilane film obtained by a spin on glass technique $^{25}$ ) and polymer film on both sides of substrate (PDA LB film). In the first case

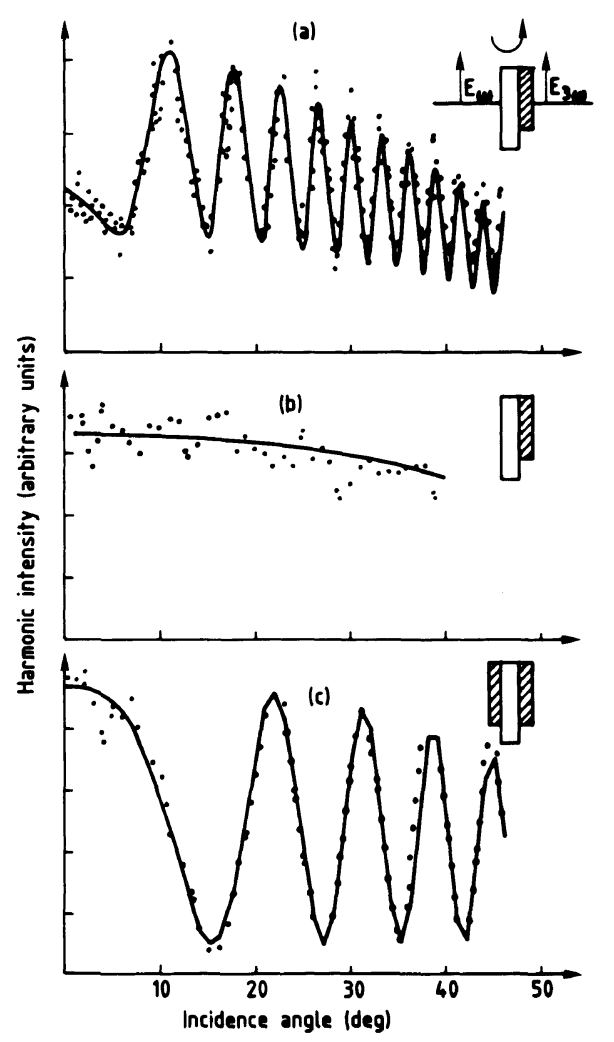

Figure 3. Third harmonic intensity in function of incidence angle from single polysilane thin film (a), a single polydiacetylene thin film (b), and from a double polydiacetylene film (c). Continuous line shows theoretical values and points measured ones. 
(Figure 3b) only harmonic light from polymer film is observed; that from substrate being negligible. For a polysilane film (see Figure 3a) an interference between harmonic field generated in substrate and in polymer film is observed (a superposition of Maker fringes from substrate with a monotonically decreasing field from polymer film as in Figure $3 \mathrm{~b}$ in function of incidence angle). This allows a $\chi^{(3)}$ phase determination. ${ }^{25}$ In Figure $3 \mathrm{c}$ fringing patterns are seen, characteristic for Maker fringes from a nonlinear slab. However origin of the present interference picture is quite different than in the case of Maker fringes. In the present case they are due to an interference between harmonic field generated in first (on one side) and in the second polymer film (on the other side) the phase mismatch, and consequently the interference being due to the refractive index dispersion in substrate, and not in nonlinear medium (polymer film) as in the former case. From Figure 3 it is seen also that theory (solid lines) fits very well experimental spectra points.

All measurements have been done in vacuum. We note here, that in air both intensity and envelope of harmonic light are modified. ${ }^{8}$ The measured intensity in air is smaller than in vacuum, the decrease depending on focal length and on incident light wavelength. The envelope function in air falls more rapidly with incidence angle in the case of smaller air contribution than signal from sample itself and increases in the opposite case. Again, these effects depend on focal length and incidence wavelength.

In Figures 4 and 5 we have displayed the chain parallel cubic susceptibility $\chi_{x x x x}^{(3)}(-3 \omega$; $\omega, \omega, \omega)$ in function of incidence wavelength for blue and red form of PDA thin films, respectively. For blue form we observe a maximum in $\chi^{(3)}$ at $1.35 \mu \mathrm{m}$ fundamental wavelength. As the wavelength is falling in the polymer transparency range, the only explication of this resonance, as it will be discussed

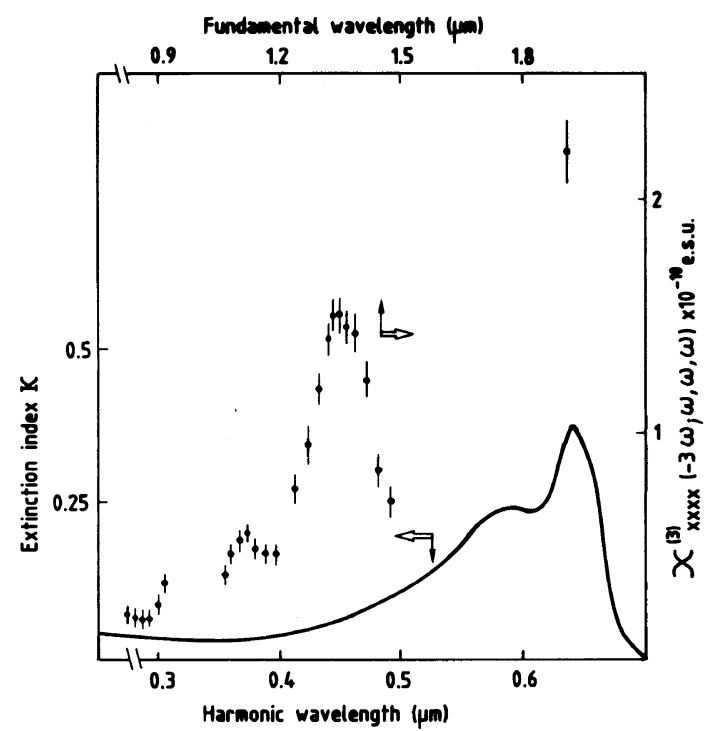

Figure 4. Cubic susceptibility $\chi_{x x x x}^{(3)}(-3 \omega ; \omega, \omega, \omega)$ in function of fundamental wavelength for blue form of polydiacetylene LB film. Solid line show imaginary part of refractive index.

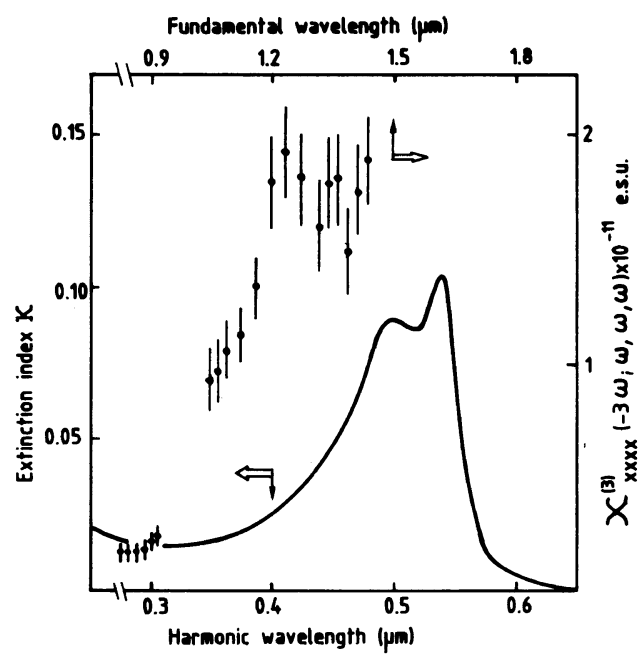

Figure 5. Cubic suscpetibility $\chi_{x x x x}^{(3)}(-3 \omega ; \omega, \omega, \omega)$ in function of fundamental wavelength for red form of polydiacetylene LB film. Other details as in Figure 4.

in next section, is via a two photon resonance with a forbidden level. The second increase in $\chi^{(3)}$ is a three photon resonance; the harmonic frequency falling at the excitonic absorption peak (continuous line). A small peak around 
$1.06 \mu \mathrm{m}$ is probably a resonance with a forbidden conduction state. In the case of red form of polymer (Figure 5) we observe also a maximum in $\chi^{(3)}(-3 \omega ; \omega, \omega, \omega)$ at about $1.22 \mu \mathrm{m}$ followed by an increase in cubic susceptibility for larger wavelength due to a three photon resonance.

\section{DISCUSSION}

In order to understand the origin of observed resonances in cubic susceptibility $\chi^{(3)}$ $(-3 \omega ; \omega, \omega, \omega)$ we use the quantum mechanical formulas obtained by time dependent perturbative calculation. For a linearly polarized incident light one obtains for the tensor component responsible for THG process in dipolar approximation following expression $^{26}$ :

$$
\begin{aligned}
\chi^{(3)}(\cdots) \alpha \sum_{n f n^{\prime}} \Omega_{g n} \Omega_{n f} \Omega_{f n^{\prime}} \Omega_{n^{\prime} g} \\
\quad \times\left[\frac{1}{\left(E_{n g}-3 \omega\right)\left(E_{f g}-2 \omega\right)\left(E_{n^{\prime} g}-\omega\right)}\right. \\
+\frac{1}{\left(E_{n g}+\omega\right)\left(E_{f g}-2 \omega\right)\left(E_{n^{\prime} g}-\omega\right)} \\
+\frac{1}{\left(E_{n g}+\omega\right)\left(E_{f g}+2 \omega\right)\left(E_{n^{\prime} g}-\omega\right)} \\
\left.+\frac{1}{\left(E_{n g}+\omega\right)\left(E_{f g}+2 \omega\right)\left(E_{n^{\prime} g}+3 \omega\right)}\right]
\end{aligned}
$$

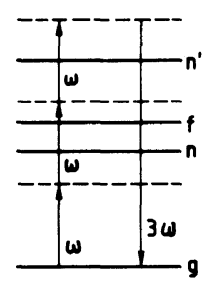

(a)

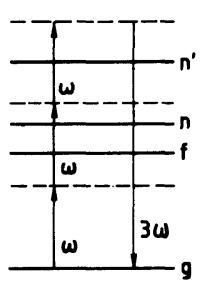

(b)
Figure 6. Optical third harmonic generation process: solid lines represent excited unperturbed levels whereas broken lines show virtual states. States $n$ and $n^{\prime}$ have opposite symmetry to the fundamental one and state $f$ the same. In studied polymers state $f$ lies below $n$ and $n^{\prime}$ as in (b). where $g$ is the fundamental state (see Figure 6), $E_{i j}$ is energy difference between states $i$ and $j$ in $\hbar$ units $\left(E_{i j}=\left(E_{i}-E_{j}\right) / \hbar\right), \Omega_{i j}$ dipolar transition matrix elements between states $i$ and $j: \Omega_{i j}=\langle j|$ er $|i\rangle$. The transition matrix elements $\Omega_{i j}$ are non zero only if $i$ and $j$ states have opposite symmetry. It means that if states $n$ and $n^{\prime}$ have opposite symmetry to the fundamental $g$, then $f$ will have the same.

Equation 17 shows that when the energy difference between fundamental and one of the excited levels approaches one or multiple incident photon energies then a resonance enhancement in $\chi^{(3)}(-3 \omega ; \omega, \omega, \omega)$ will occur. In THG process one, two and three photon resonances are possible. One photon resonance is possible when the fundamental wavelength falls in the absorption region. Such a resonance enhancement in $\chi^{(3)}$ was observed in PDA LB film by Carter et al. ${ }^{27}$

Three photon resonance $\left(E_{n g}-3 \omega=0\right)$ was observed by Sauteret et al. ${ }^{28}$ in poly(toluene sulphonate) (PTS) single crystals, in blue PDA LB film ( $c f$. Figure 4) and in trans-polyacetyl$e^{2 n} e^{29}$; all at $1.907 \mu \mathrm{m}$ fundamental wavelength. A three photon resonance was also observed in polysilane thin films at $1.064 \mu \mathrm{m} .^{25}$ The two photon resonance was first observed in PDA LB film ${ }^{18}$ (cf. Figure 4) at $1.35 \mu \mathrm{m}$. Similarly, it was also observed in trans- $(\mathrm{CH})_{x}$ thin film at the same wavelength ${ }^{29}$ and at 1.22 in red PDA LB film ( $c f$. Figure 5).

We note here that in both cases red and blue form in PDA, as well as in trans- $(\mathrm{CH})_{x}$ the double of the incident photon energy at two photon resonance is smaller than the gap energy. It means that in these conjugated polymers the forbidden two photon level lies below the allowed for optical transition $B_{\mathrm{u}}^{1}$ state ( $c f$. Figure $6 \mathrm{~b}$ ) $2500 \mathrm{~cm}^{-1}, 800 \mathrm{~cm}^{-1}$ and $900 \mathrm{~cm}^{-1}$ in PDA red, PDA blue and transPA, respectively. From a practical point of view one and two photon resonances have little perspectives of applications in optical devices. This is connected with fundamental beam absorption in first case, resulting in thin film 
heating. In the case of three photon resonance the only possibility of its application is frequency conversion. Due to the fact that the harmonic light is strongly absorbed (it falls at absorption peak), only a small interaction length can be used, resulting in limited conversion efficiency.

The two photon resonance may have much more practical impact than one and three photon resonances. It may be used in the frequency conversion. A priori there is no limitation on fundamental beam power; this one falling at the polymer transparency region. Also the harmonic frequency ( $c f$. Figure 4) is not strongly absorbed (it falls in the deep of polymer absorption). However at the two photon resonance the cubic susceptibility is complex and its real and imaginary parts have following form (in a three level approximation: $n=n^{\prime} \neq f$ ).

$$
\operatorname{Re} \chi^{(3)}(-3 \omega ; \omega, \omega, \omega) \alpha \frac{E_{f g}-2 \omega}{\left(E_{f g}-2 \omega\right)^{2}+\Gamma^{2}} A
$$

and

$$
\operatorname{Jm} \chi^{(3)}(-3 \omega ; \omega, \omega, \omega) \frac{\Gamma}{\left(E_{f g}-2 \omega\right)^{2}+\Gamma^{2}} A
$$

where

$$
A=\sum_{n, n^{\prime}} \frac{\Omega_{g n} \Omega_{n f} \Omega_{f n^{\prime}} \Omega_{n^{\prime} g}}{E_{n^{\prime} g}-\omega}\left[\frac{1}{E_{n g}-3 \omega}+\frac{1}{E_{n g}+\omega}\right]
$$

It means that at two photon resonance the real part of $\chi^{(3)}$ is equal to zero and the imaginary part reaches its maximum. At the same time the Kerr susceptibility $\chi^{(3)}(-\omega$; $\omega, \omega,-\omega)$ will be also complex with a similar frequency behavior as $\chi^{(3)}(-3 \omega ; \omega, \omega, \omega)$ (see eq 18-19. Complex will be also intensity dependent index of refraction given by:

$$
n=n_{0}+n_{2} J_{\omega}
$$

where $n_{0}$ is linear refractive index and

$$
n_{2}=12 \pi^{2} \chi^{(3)}(-\omega ; \omega, \omega,-\omega) / n_{0}^{2} c
$$

It follows that the fundamental beam at frequency $\omega$ will be absorbed via a two photon process (two photon absorption-TPA) reaching a maximum at two photon resonance exactly and resulting in a pump depletion. It is possible to diminish this absorption by a slight shift of the pump frequency beyond two photon resonance (e.g., $\omega_{p}=\omega_{f g} \pm \Gamma$ where $\Gamma$ is two photon level width).

The two photon absorption and resulting refractive index variation with incident power will lead to an active optical bistability with a large number of possible applications (amplification, optical transistors, dynamic memories etc.).

For blue form of PDA, assuming that the resonant value of $\chi^{(3)}(-\omega ; \omega,-\omega, \omega)$ is close to that of $\chi^{(3)}(-3 \omega ; \omega, \omega, \omega)$ one gets $n_{2} \simeq 10^{-6}$ $\mathrm{cm}^{2} / \mathrm{MW}$. Under the same assumption one obtains an order of magnitude larger value for trans $-(\mathrm{CH})_{x}$. These are large numbers comparable or larger than in inorganic semiconductors in the same frequency range.

Similarly, two photon resonance may be also used in optical and electro-optical switches operating at $1.35 \mu \mathrm{m}$.

\section{CONCLUSIONS}

In this paper we have described our recent work on nonlinear optical properties of conjugated polymers. The measured values of $\chi_{x x x x}^{(3)}(-3 \omega ; \omega, \omega, \omega)$, corrected for polymer disorder by a factor given by

$$
\left\langle\cos ^{4} \theta\right\rangle=\frac{3}{8}
$$

where $\theta$ is angle between polymer chain and incident electric field, are listed in Table I together with other reference values. The polymer $\chi^{(3)}$ values were calibrated with fused silica in vacuum $\chi_{x x x x}^{(3)}=2.8 \times 10^{-14}$ e.s.u. ${ }^{11}$ This shows that the conjugated polymers are highly polarizable, with highest observed values for $\chi^{(3)}(-3 \omega ; \omega, \omega, \omega)$ in the case of trans- $(\mathrm{CH})_{x}$. This make them an interesting 
Table I. Cubic susceptibilities $\chi_{x x x x}^{(3)}(-3 \omega ; \omega, \omega, \omega)$ in $10^{-14}$ e.s.u. of conjugated polymers and other related materials at different wavelengths

\begin{tabular}{|c|c|c|c|}
\hline \multirow{2}{*}{ Material } & \multicolumn{3}{|c|}{$\chi_{x x x x}^{(3)}(-3 \omega ; \omega, \omega, \omega)\left(\times 10^{-14}\right.$ e.s.u. $)$} \\
\hline & $1.064 \mu \mathrm{m}$ & $1.35 \mu \mathrm{m}$ & $1.907 \mu \mathrm{m}$ \\
\hline Polydiacetylene LB film flue form ${ }^{a}$ & $(3.4 \pm 0.3) \times 10^{3}$ & $(1.5 \pm 0.2) \times 10^{4}$ & $(2.2 \pm 0.2) \times 10^{4}$ \\
\hline Polydiacetylene LB film red form ${ }^{b}$ & $(0.9 \pm 0.1) \times 10^{3}$ & $(1.9 \pm 0.2) \times 10^{3}$ & $(3.4 \pm 0.3) \times 10^{3}$ \\
\hline trans-Polyacetylene ${ }^{c}$ & $(4 \pm 0.5) \times 10^{3}$ & $(1.2 \pm 0.1) \times 10^{5}$ & $(1.3 \pm 0.1) \times 10^{5}$ \\
\hline Poly-TS- $6^{d}$ & & & $(8.5 \pm 5) \times 10^{4}$ \\
\hline TCDU $^{d}$ & & & $(7 \pm 5) \times 10^{3}$ \\
\hline Polysilane $^{\mathrm{e}}$ & $(1.5 \pm 0.1) \times 10^{2}$ & & \\
\hline Benzene $^{f}$ & $12.0 \pm 0.6$ & & $10.6 \pm 0.5$ \\
\hline Silica ${ }^{\mathrm{g}}$ & & & 2.8 \\
\hline
\end{tabular}

${ }^{a} \operatorname{Ref} 18 .{ }^{\mathrm{b}} \operatorname{Ref} 32 .^{\mathrm{c}} \operatorname{Ref} 29 .^{\mathrm{d}}$ Single crystal value (ref 28). ${ }^{\mathrm{e}}$ Average value $\left\langle\chi^{(3)}(-3 \omega ; \omega, \omega, \omega)\right\rangle$ (ref 25). ${ }^{\mathrm{f}}$ Ref $8 .{ }^{\mathrm{g}}$ Ref 11.

candidate for applications in optical devices.

The discovery of two photon resonance at $1.35 \mu \mathrm{m}$ in blue form of PDA and in trans$(\mathrm{CH})_{x}$ make them especially interesting for applications in telecommunications; the above wavelength falling in the best optical fibers transparency region.

The ease in thin film preparation by different techniques, possibility of circuits design by solid state polymerization or photodecomposition show that conjugated polymers are promissing candidates for applications in integrated optics. ${ }^{30}$ The EFISHG experiments show two phenomena. ${ }^{14,31}$ First one is a broad two photon resonance around the same wavelength as in THG experiments. The broadening is due to the fact that in EFISHG the two photon resonance occur simultaneously with allowed and forbidden ( $n$ and $f$ states in Figure 6) for one photon transition levels ( $c f$. discussion in Chollet $e t$ $\left.a l^{32}\right)$. As these levels are close, the resonance width is broaded.

The second phenomenon is connected with a large internal polarization creation by a two photon absorption process for incident wavelength smaller than $1.1 \mu \mathrm{m}$. As incident photon energy is smaller than the band to band transition energy, the electron-hole pair generation is only possible with two photon process. This may be done primarily via a two photon absorption or by a reabsorption of generated photons with double frequency (SHG). The so created electron-hole pairs are separated by applied DC field and trapped by polymer defects, creating a large internal polarization. This separation is large in LB films and internal polarization reaches the values of the order of $5 \times 10^{4} \mathrm{~V} \mathrm{~cm}^{-1}$. This polarization can be erazed by a visible or again IR light through one or two photon process, respectively.

Acknowledgements. The authors would like to express their gratitude to direction des Affaires Industrielles et Internationales (Ministère des PTT) for a partial financial support of this work.

\section{REFERENCES}

1. K. C. Rustagi and J. Ducuing, Opt. Commun., 10, 258 (1974).

2. G. P. Agrawal, C. Cojan, and C. Flytzanis, Phys. Rev̀., B17, 776 (1978).

3. D. N. Batchelder and D. Bloor, J. Polym. Sci., Polym. Phys. Ed., 17, 569 (1981).

4. R. H. Baughman, H. Gleiter, and N. Sendfeld, $J$. Polym. Sci., Polym. Phys. Ed., 13, 1871 (1975).

5. B. Tieke, H. J. Graf, G. Wegner, B. Naegele, H. Ringsdorf, A. Banerjee, D. Day, and J. B. Lando, Colloid. Polym. Sci., 255, 521 (1977). 


\section{F. KaJZAR and J. Messier}

6. S. E. Rickert, J. B. Lando, and D. Ching, "Nonlinear Optical Properties of Organic and Polymeric Materials," D. J. Williams, Ed., American Chemical Society Symposium Series, No. 233, Washington D.C., 1983, p 229.

7. M. Thakur and S. Meyer, Macromolecules, 18, 2341 (1985).

8. F. Kajzar and J. Messier, Phys. Rev. A, 32, 2352 (1985).

9. J. Jerphagnon and S. K. Kurtz, J. Appl. Phys., 41, 1667 (1970); Phys. Rev. B1, 1739 (1970).

10. J. L. Oudar, J. Chem. Phys., 67, 446 (1977).

11. G. R. Meredith, B. Buchalter, and C. Hanzlik, J. Chem. Phys., 78, 1533 (1983); ibid., 78, 1543 (1983).

12. F. Kajzar and J. Messier, "Nonlinear Optical Properties of Organic Materials and Crystals," Vol. 2, D. S. Chemla and J. Zyss, Ed., Academic Press, Chapter III-2, in press.

13. F. Kajzar, J. Messier, and J. Zyss, J. Phys., 44, C3709 (1983).

14. P-A. Chollet, F. Kajzar, and J. Messier, Thin Solid Films, 132, 1 (1985).

15. M. Choy and R. L. Byer, Phys. Rev., B14, 1693 (1976).

16. G. Wegner, Faraday Disc. Chem. Soc., 68, 494 (1980).

17. D. Day and J. B. Lando, Macromolecules, 13, 1478 (1980).

18. F. Kajzar and J. Messier, Thin Solid Films, 132, 11 (1985).

19. G. Lieser, B. Tieke, and G. Wegner, Thin Solid Films,
68, 77 (1980).

20. P. A. Chollet, Thin Solid Films, 68, 13 (1980).

21. F. Kajzar and J. Messier, Chem. Phys., 63, 123 (1981).

22. B. Belbeoch, M. Roulliay, and M. Tournarie, Thin Solid Films, 134, 89 (1985).

23. A. Bonnerot, P. A. Chollet, M. Frisby, and M. Hoclet, Chem. Phys., 97, 365 (1985).

24. R. R. Highfield, R. K. Thomas, P. G. Cummins, D. P. Gregory, J. Mingins, J. B. Hayer, and O. Scharpf, Thin Solid Films, 99, 165 (1983).

25. F. Kajzar, J. Messier, and C. Rosilio, J. Appl. Phys., 60, 3040 (1986).

26. D. C. Hanna, M. A. Yuratich, and D. Cotter, Nonlinear Optics of Free Atoms and Molecules," Springer Verlag, Berlin and New York, 1979.

27. G. M. Carter, M. K. Thakur, Y. J. Chen, and J. V. Hryniewin, Appl. Phys. Lett., 47, 457 (1985).

28. C. Sauteret, J. P. Hermann, R. Frey, F. Pradere, R. H. Baughman, and R. R. Chance, Phys. Rev. Lett., 36, 956 (1976).

29. F. Kajzar, S. Etemad, G. L. Baker, and J. Messier, Synthetic Metals, in print.

30. J. Zyss, J. Mol. Electronics, 1, 25 (1985).

31. P. A. Chollet, F. Kajzar, and J. Messier, "Polydiacetylenes," D. Bloor and R. R. Chance, Ed., NATO ASI Series, No. E102, Martinus Nijhoff Publishers, Dordrecht, 1985, p 317.

32. P. A. Chollet, F. Kajzar, and J. Messier, Synthetic Metals, in print. 